in die graphische Darstellung der Abb. 4 mit aufgenommen. Die Übereinstimmung der nach dieser Methode ermittelten Diffusionskoeffizienten mit den aus den stationären Messungen und den Wasserstofflöslichkeiten berechneten Diffusionskoeffizienten ist ein weiterer Hinweis dafür, daß die Kinetik der Permea- tion allein durch die Volumendiffusion bestimmt wird.

Im gleichen Sinne läßt sich schließlich der Befund deuten, daß die im technischen Wasserstoff enthaltenen Verunreinigungen keinen Einfluß auf die Permeation haben.

\title{
Die solaren Bursts vom Typ III
}

\author{
R. WAGNER \\ Institut für Theoretische Physik und Sternwarte der Universität Kiel \\ (Z. Naturforschg. 22 a, 1586-1592 [1967] ; eingegangen am 14. März 1967)
}

\begin{abstract}
In a previous paper formulae for the radio emission originating from the scattering of a plane plasma electron wave by thermal fluctuations were derived. These formulae are applied to the solar type III bursts by starting from a cloud of electrons which trausverses the solar corona thereby exciting plasma waves. The electric field of the excited waves is estimated by an expression of the quasilinear theory. The band width of these waves is about $10 \%$ of the local plasma frequency. The scattering mechanism considered here can account only for the faint and moderate type III bursts without harmonic structure. The possibility to determine the thickness of the cloud in its variation with time is discussed.
\end{abstract}

In der vorangegangenen Arbeit *, die im folgenden mit I bezeichnet wird, wurde die Entstehung von Radiostrahlung in einem Plasma durch Streuung einer ebenen Plasmawelle an den thermischen Dichtefluktuationen des Plasmas diskutiert. Dazu wurden die Fluktuationen durch thermische Plasmaelektronen- und -ionenwellen dargestellt. Im Falle der Streuung an den thermischen Ionenwellen ergab sich eine Radioemission bei der Elektronenplasmafrequenz $\omega_{\mathrm{p}}$, während bei der Streuung an den thermischen Elektronenwellen Radiostrahlung bei $2 \omega_{\mathrm{p}}$ entstand. Die in I hergeleiteten Intensitätenformeln (92) und (63) werden im folgenden auf die Bursts vom Typ III angewandt. Dabei wird von der Tatsache ausgegangen, daß der Typ III durch Teilchenwolken hervorgerufen wird ${ }^{1,2}$. Diese Teilchenwolken müssen aus Elektronen bestehen. Die großen Stoßzeiten für Protonen in der Korona könnten die untere Grenze der radialen Geschwindigkeiten der Wolke $^{3}$ von $0,2 c \quad(c=$ Lichtgeschwindigkeit $)$ nicht erklären ${ }^{4,5}$.

Die Entstehung der Typ III-Radiostrahlung hat man sich nach WILD, SMerd und WeIss ${ }^{1}$ so vorzustellen: Die Elektronenwolke erregt über die Zwei-

* R. WAGNER, Z. Naturforschg. 22 a, 1372 [1967].

1 J. P. Wild, F. S. Smerd u. A. A. Weiss, Ann. Rev. Astron. Astrophys. 1, 291 [1963].

2 Y. Uchida, Publ. Astron. Soc. Japan 12, 376 [1960].

3 J. P. Wild, K. U. Sheridan u. A. A. Neylan, Australian J. Phys. 12, 369 [1959]. strominstabilität in der Korona Plasmawellen, die zum Teil in Radiowellen umgesetzt werden. Zur Berechnung der abgestrahlten Intensität aus (92) bzw. (93) und (63) benötigt man die elektrische Feldstärke dieser Plasmawellen.

\section{Elektrische Feldstärke der Plasmawellen}

Für ein stoßfreies Elektronenplasma mit verschmiertem Ionenhintergrund läßt sich nach KNORR ${ }^{6}$ die elektrische Feldstärke $E_{\mathrm{k}}$ einer Plasmawelle der Wellenzahl $k$ mit Hilfe der quasilinearen Theorie abschätzen. Setzt man die entsprechenden Größen des vorliegenden Problems ein, so erhält man unter der Voraussetzung, daß das System Korona - Wolke hinreichend gut durch eine Temperatur, die Koronatemperatur $T_{\mathrm{c}}$, beschrieben wird:

$$
\frac{E_{k}^{2}}{8 \pi}=\frac{n_{\mathrm{c}}}{2}(1+\mu)^{1,5} \varkappa T_{\mathrm{c}} \alpha \frac{\gamma^{3}}{k \lambda_{\mathrm{Dc}}}
$$

mit

$$
\begin{aligned}
\gamma=\frac{\pi}{2 k \lambda_{\mathrm{Dc}}}(1+\mu)^{-1 / 2}\left(\frac{\omega_{\mathrm{pc}}}{\omega}\right)^{2} \\
\cdot\left[v v_{\mathrm{Tc}}\left\{\frac{\partial f_{\mathrm{c}}}{\partial v}+\mu \frac{\partial f_{\mathrm{w}}}{\partial v}\right\}\right]_{v=\omega^{\prime} k} .
\end{aligned}
$$

4 C. DE JAGER, Space Research, Vol. I, ed. H. Kallmann Bijl, North Holland Publishing Co., Amsterdam 1960.

5 M. R. Kundu, Solar Radio Astronomy, Interscience Publishers, New York 1965.

6 G. KNORR, Z. Naturforschg. 18 a, 1304 [1963]. 
Dabei sind $n_{\mathrm{c}}$ die Elektronendichte, $\lambda_{\text {De }}$ die DeBYeLänge, $\omega_{\mathrm{pc}}$ die Plasmafrequenz, $v_{\mathrm{Tc}}$ die thermische Elektronengeschwindigkeit in der Korona. Weiter ist $\mu=n_{\mathrm{w}} / n_{\mathrm{c}}$, wobei $n_{\mathrm{w}}$ die Elektronendichte in der Wolke ist, $\varkappa$ die Boltzmann-Konstante, $\omega$ die Kreisfrequenz der Plasmawelle. $\gamma$ ist die dimensionslose Anwachsrate der Zweistrominstabilität. Für den Proportionalitätsfaktor $\alpha$ ergibt sich durch direkte Integration der Vlasov-Gleichung ${ }^{6} \alpha \approx 3$. Für die normierte Elektronenverteilungsfunktion in der Korona, $f_{\mathrm{c}}$, bzw. in der Wolke, $f_{\mathrm{w}}$, wird angesetzt:

$$
f_{\mathrm{c}}(v)=(2 \pi)^{-1 / 2} v_{\mathrm{Tc}}^{-1} \exp \left(-v^{2} / 2 v_{\mathrm{Tc}}^{2}\right) \text {, }
$$

$$
f_{\mathrm{w}}(v)=(2 \pi)^{-1 / 2} v_{\mathrm{Tc}}^{-1} \vartheta^{-1 / 2} \exp \left(-\frac{\left(v-v_{0} \cos \eta\right)^{2}}{2 \vartheta v_{\mathrm{Tc}}^{2}}\right) .
$$

Darin ist $\vartheta=T_{\mathrm{w}} / T_{\mathrm{c}}$, wobei $T_{\mathrm{w}}$ die Temperatur der Wolke ist, $v_{0}=\left|\mathfrak{b}_{0}\right|$ die Geschwindigkeit der Wolke und $\eta$ der Winkel zwischen der Fortpflanzungsrichtung der betrachteten Plasmawelle und $\mathfrak{v}_{0}$. In $f_{\mathrm{w}}$ tritt also nur eine "effektive“ Geschwindigkeit $v_{0} \cos \eta$ auf, da bei der Herleitung von $\gamma$, wie in allen ähnlichen Rechnungen mit Plasmawellen, die Verteilungsfunktion über die zu $k$ senkrechte Geschwindigkeitskomponente integriert wurde. Mit (3) folgt

$$
\begin{aligned}
\gamma=-\frac{\pi}{2 k \lambda_{\mathrm{Dc}}}(1+\mu)^{-1 / 2}(2 \pi)^{-1 / 2}[ & \frac{1}{\left(k \lambda_{\mathrm{Dc}}\right)^{2}} \exp \left(-\frac{\omega^{2}}{2 k^{2} v_{\mathrm{Tc}}^{2}}\right) \\
& \left.+\frac{\mu}{\vartheta} \frac{1}{k \lambda_{\mathrm{Dc}}} \frac{\omega_{\mathrm{pc}}}{\omega} \frac{\omega / k-v_{0} \cos \eta}{\sqrt{\vartheta} v_{\mathrm{Tc}}} \exp \left\{-\frac{\left(\omega / k-v_{0} \cos \eta\right)^{2}}{2 \vartheta v_{\mathrm{Tc}}^{2}}\right\}\right] .
\end{aligned}
$$

Da das erste Glied in (4) wegen seiner Kleinheit vernachlässigt werden kann, gilt:

$$
\gamma=\frac{1}{2} \sqrt{\frac{\pi}{2}}(1+\mu)^{-1 / 2} \mu\left(\frac{\omega_{\mathrm{pc}}}{\omega}\right)^{3}\left(\frac{\omega / k}{\sqrt{ } \vartheta v_{\mathrm{Tc}}}\right)^{2} \frac{v_{0} \cos \eta-\omega / k}{\sqrt{\vartheta} v_{\mathrm{Tc}}} \cdot \exp \left\{-\frac{\left(\omega / k-v_{0} \cos \eta\right)^{2}}{2 \vartheta v_{\mathrm{Tc}}^{2}}\right\} .
$$

Zur Abkürzung schreiben wir

\begin{tabular}{|c|c|c|c|c|c|}
\hline$y$ & 3,2 & 5,8 & 10,9 & 20,95 & 51,0 \\
\hline$x_{\max }$ & 2,5 & 5 & 10 & 20 & 50 \\
\hline $5 \mu^{-1}(1+\mu)^{1 / 2} \gamma_{\max }$ & 2,1 & 8,9 & 36,9 & 148,4 & 929,6 \\
\hline
\end{tabular}
$\omega\left(k \sqrt{\vartheta} v_{\mathrm{Tc}}\right)^{-1}=x$ und $v_{0} \cos \eta\left(\sqrt{\vartheta} v_{\mathrm{Tc}}\right)^{-1}=y$. Setzen wir noch $\omega_{\mathrm{pc}} / \omega \approx 1$, so erhalten wir für die von $y$ abhängigen, bei $x=x_{\max }$ angenommenen maximalen Anwachsraten $\gamma_{\max }$ die Werte der Tab. 1 .

Tab. 1. Die bei $x_{\max }=(\omega / k V \bar{\vartheta} v \mathrm{Tc})_{\max }$ angenommene dimensionslose maximale Anwachsrate $\gamma_{\max }$ als Funktion von $y=v_{0} \cos \eta\left(\sqrt{\vartheta} v_{\mathrm{Tc}}\right)^{-1}$.

Nach Stewart ${ }^{7}$ ist die Geschwindigkeit $v_{0}$ der Wolke im Mittel $v_{0}=\frac{1}{3} c$. Mit $v_{\mathrm{Tc}}=5,5 \cdot 10^{8} \mathrm{~cm} / \mathrm{sec}$ folgt daraus, solange $\vartheta \approx 1$ gilt, $y \leqq 20$, also ein mittlerer Wert $\gamma_{\max } \approx 50(1+\mu)^{-1 / 2} \mu$. Damit liefert Gl. (1) :

$$
E_{k}^{2} / 8 \pi=1,9 \cdot 10^{5} n_{\mathrm{c}} \varkappa T_{\mathrm{c}} \mu^{3} / k \lambda_{\mathrm{Dc}} .
$$

Die elektrische Energiedichte der von der Wolke über die Zweistrominstabilität angeregten Plasmawellen hängt also stark von $\mu=n_{\mathrm{w}} / n_{\mathrm{c}}$ und damit der Elektronendichte in der Wolke und schwächer von der Wellenzahl $k$ der angeregten Wellen ab.

\section{Spektrale Breite der Plasmawellen}

Im Zusammenhang mit der Abhängigkeit der elektrischen Energiedichte von der Wellenzahl $k$ in Gl. (6) stellt sich die Frage, welche Wellenzahlen die in einem bestimmten Niveau der Sonnenkorona durch die Wolke angeregten Plasmawellen besitzen können, d.h. wie groß die spektrale Breite der in einem bestimmten Niveau angeregten Plasmawellen ist. Aus Gl. (5) ersieht man unmittelbar, daß das System Wolke-Korona nur für solche Plasmawellen instabil sein kann, deren Phasengeschwindigkeit $\omega / k$ kleiner ist als die „effektive“ Wolkengeschwindigkeit $v_{0} \cos \eta$. (Sonst wäre $\gamma<0$.) Damit ist die minimale Wellenzahl $k_{\min }$ durch die Bedingung $\omega / k_{\min }=v_{0}$ gegeben. Mit der Dispersionsrelation für Plasmawellen und $v_{0}=\frac{1}{3} c$ folgt

$$
\left(k \lambda_{\mathrm{Dc}}\right)_{\min }=5,5 \cdot 10^{-2}, \quad \omega_{\min }=1,0045 \omega_{\mathrm{pc}} .
$$

Die maximale Wellenzahl $k_{\max }$ ergibt sich aus den Rechnungen von $\mathrm{J}_{\mathrm{ACKSON}}{ }^{8}$, in denen die Instabilität zweier sich durchdringender Plasmen mit MaxwellVerteilungen betrachtet wurde. Die Ergebnisse der zitierten Arbeit können wir übernehmen, wenn wir die Korona als Elektronenplasma mit verschmiertem Ionenhintergrund ansehen [wie wir das schon zur

8 E. A. JACKson, Phys. Fluids 3, 786 [1960]. 
Herleitung von Gl. (6) getan haben]. Für $k_{\max }$, die zugehörende Kreisfrequenz $\omega_{\max }$ und die Relativgeschwindigkeit Wolke-Korona (wieder die effektive!), für die die Plasmawellen maximaler Wellenzahl angeregt werden, ergeben sich in Abhängigkeit von $\left(\lambda_{\mathrm{Dc}} / \lambda_{\mathrm{Dw}}\right)^{2}\left(\lambda_{\mathrm{Dw}}=\right.$ DeBye-Länge in der Wolke $)$ die Werte der Tab. 2 .

\begin{tabular}{clllll}
\hline$\left(\lambda_{\mathrm{Dc}} / \lambda_{\mathrm{Dw}}\right)^{2}$ & \multicolumn{1}{c}{1} & 0,25 & $10^{-1}$ & $10^{-2}$ & $10^{-3}$ \\
$\left(k \lambda_{\mathrm{Dc}}\right)_{\max }$ & 0,755 & 0,55 & 0,46 & 0,28 & 0,24 \\
$\left(\omega / \omega_{\mathrm{pc}}\right)_{\max }$ & 1,60 & 1,40 & 1,33 & 1,11 & 1,08 \\
$u / v_{\mathrm{Tc}}$ & $2,12 \sqrt{\vartheta}$ & $1,70 \sqrt{\vartheta}$ & $1,50 \sqrt{\vartheta}$ & $0,99 \sqrt{\vartheta}$ & $0,99 \sqrt{\vartheta}$ \\
& $+2,12$ & $+2,55$ & $+2,87$ & $+3,57$ & $+4,17$ \\
\hline
\end{tabular}

Tab. 2. Relativgeschwindigkeit $u$, bei der die Plasmawelle mit maximaler Wellenzahl $k_{\max }$ bzw. maximaler Kreisfrequenz $\omega_{\max }$ angeregt wird, in Abhängigkeit von $\left(\lambda_{\mathrm{Dc}} / \lambda_{\mathrm{Dw}}\right)^{2}$.

Die ersten 3 Spalten der Tab. 2 ergeben sich aus den Tabellen der zitierten Arbeit, die letzten beiden aus den dort angegebenen asymptotischen Formeln. Aus der Tabelle ist ersichtlich, daß die Relativgeschwindigkeit $u$, bei der die Plasmawelle maximaler Wellenzahl abgestrahlt wird, in der Größenordnung der thermischen Elektronengeschwindigkeit $v_{\mathrm{Tc}}$ in der Korona liegt, solange $\vartheta \lesssim 1$, was im folgenden wieder angenommen wird. Andererseits durchläuft die "effektive" Geschwindigkeit $v_{0} \cos \eta$ wegen $0 \leqq \eta \leqq \frac{1}{2} \pi$ alle Werte $<v_{0}$. Das bedeutet wegen $v_{0} \gg v_{\mathrm{Tc}}$ : In einem bestimmten Niveau der Sonnenkorona werden Plasmawellen angeregt in einem großen Kegel um die Geschwindigkeitsrichtung der Wolke. Die angeregten Wellen besitzen Wellenzahlen zwischen $k_{\min }$ und $k_{\max }$, wobei $k_{\min }$ durch (7) gegeben ist und $k_{\max }$ gemäß Tab. 2 vom Verhältnis $\left(\lambda_{\mathrm{Dc}} / \lambda_{\mathrm{Dw}}\right)^{2}$ abhängt. Berücksichtigt man noch die Gleichung

$$
\left(\lambda_{\mathrm{Dc}} / \lambda_{\mathrm{Dw}}\right)^{2}=\mu / \vartheta,
$$

so folgt, daß die spektrale Breite der angeregten Plasmawellen von physikalischen Größen der Wolke selbst, nämlich der Elektronendichte und der Temperatur abhängt.

\section{Die Elektronendichte der Wolke}

Wir fragen nach der Bestimmung von $\mu=n_{\mathrm{w}} / n_{\mathrm{c}}$ und $\vartheta=T_{\mathrm{w}} / T_{\mathrm{c}}$. Ein Weg zur größenordnungsmäßigen Abschätzung von $\mu$ besteht darin, die elektrische Energiedichte aus (6) in eine theoretische Intensitätenformel einzusetzen und $\mu$ dann so zu bestimmen, daß sich die beobachteten Intensitäten er- geben. Im folgenden wollen wir zunächst nur die Grundfrequenz betrachten; daher gehen wir von Gl. (92) der Arbeit I aus. Diese Formel schreiben wir auf den Strahlungsstrom $F$ (pro $\mathrm{Hz}$ ) in Erdnähe um. Ist $\Delta v$ die beobachtete Bandbreite der elektromagnetischen Strahlung und $R$ der mittlere Abstand Erde-Sonne, so folgt aus Gl. (92) der Arbeit I:

$$
\begin{aligned}
F=\frac{1}{48 \pi^{2}} & \frac{1}{R^{2}} \frac{E_{k}^{2}}{8 \pi} V \frac{\omega_{\mathrm{pc}}^{2}}{c^{3} n_{\mathrm{c}}} \\
& \cdot\left(1-\frac{\Omega_{\mathrm{pc}}^{2}}{\left(\Omega+\Omega_{1}\right)^{2}}\right) \frac{1}{\Delta v} \frac{\left(k \lambda_{\mathrm{Dc}}\right)^{2}+1}{\left(k \lambda_{\mathrm{Dc}}\right)^{2}+2} .
\end{aligned}
$$

Dabei ist

$$
\Omega_{\mathrm{pc}}=\omega_{\mathrm{pc}} \sqrt{1+m / M},
$$

wobei $m$ die Elektronenmasse, $M$ die Protonenmasse ist. $\Omega_{1}$ ist die Kreisfrequenz der Plasmawelle, die gestreut wird. $\Omega$ ist eine gewisse Frequenz aus dem Spektrum der thermischen Plasmaionenwellen (siehe Arbeit I), und es gilt: $\Omega \ll \Omega_{1}$. $V$ ist das emittierende Plasmavolumen. (93) aus I trägt ebenfalls zur Emission in der Grundfrequenz mit etwa derselben Stärke bei. Der Frequenzunterschied zu (92) aus I ist dabei so klein, daß er durch die spektrale Breite der Plasmawellen verwischt wird. Mit (6) folgt, wenn wir zur Berücksichtigung von Gl. (93) aus I noch einen Faktor 2 hinzufügen:

$$
\begin{aligned}
& F=8 \cdot 10^{2} \varkappa T_{\mathrm{c}} \frac{V}{R^{2}} \frac{\omega_{\mathrm{pc}}^{4}}{c^{3}} \frac{\mu^{3}}{k \lambda_{\mathrm{Dc}}} \\
& \cdot\left(1-\frac{\Omega_{\mathrm{pc}}^{2}}{\left(\Omega+\Omega_{1}\right)^{2}}\right) \frac{1}{\Delta v} \frac{\left(k \lambda_{\mathrm{Dc}}\right)^{2}+1}{\left(k \lambda_{\mathrm{Dc}}\right)^{2}+2} .
\end{aligned}
$$

Bei einem möglichen Bereich für $k \lambda_{\text {Dc }}$ von $5,5 \cdot 10^{-2}$ $\lesssim k \lambda_{\mathrm{Dc}} \lesssim 0,75$ folgt aus Tabelle 2 der Arbeit I

$$
\left[1-\Omega_{\mathrm{pc}}^{2} /\left(\Omega+\Omega_{1}\right)^{2}\right]\left(1 / k \lambda_{\mathrm{Dc}}\right) \approx 0,3 .
$$

Außerdem ist $\quad\left[\left(k \lambda_{\mathrm{Dc}}\right)^{2}+1\right] /\left[\left(k \lambda_{\mathrm{Dc}}\right)^{2}+2\right] \approx 1$.

Aus Beobachtungen von Hughes und Harkness ${ }^{9}$ entnimmt man bei $100 \mathrm{MHz} \Delta v \approx v_{\mathrm{c}}$, wobei $\omega_{\mathrm{pc}}=2 \pi v_{\mathrm{c}}$ ist, und erhält nach Einsetzen der Konstanten:

$$
F=0,9 \cdot 10^{-47} \nu_{\mathrm{c}}^{3} \mu^{3} T_{\mathrm{c}} V\left[\mathrm{~W} \mathrm{~m}^{-2} \mathrm{~Hz}^{-1}\right] .
$$

Dabei ist $\nu_{\mathrm{c}}$ in $\mathrm{MHz}, T_{\mathrm{c}}$ in $10^{6}{ }^{\circ} \mathrm{K}, V$ in $\mathrm{cm}^{3}$ einzusetzen. Weiter ist $V=A \cdot d$, wobei $A$ die Fläche der Typ III $\left(\approx 2 \cdot 10^{20} \mathrm{~cm}^{2}\right.$, entsprechend einem Winkeldurchmesser von $4^{\prime}$ bei $100 \mathrm{MHz}, \mathrm{KuNDU}^{5}$ ) und $d$ die Dicke der Elektronenwolke $\left(\approx 10^{10} \mathrm{~cm}\right.$, Hughes und Harkness ${ }^{9}$ ) bezeichnet. Wählen wir

9 M. P. Hughes u. R. L. Harkness, Astrophys. J. 138, 239 [1963]. 
$v_{\mathrm{c}}=100 \mathrm{MHz}, T_{\mathrm{c}}=2 \cdot 10^{6}{ }^{\circ} \mathrm{K}$, so folgt

$$
F=3,6 \cdot 10^{-11} \mu^{3}\left[\mathrm{~W} \mathrm{~m}^{-2} \mathrm{~Hz}^{-1}\right] .
$$

Dieser theoretische Strahlungsstrom ist mit dem bei $100 \mathrm{MHz}$ gemessenen zu vergleichen. Dazu unterscheiden wir mit Swarup, Stone und Maxwell ${ }^{10}$ schwache Bursts:

$$
F=(5-40) \cdot 10^{-22} \mathrm{~W} \mathrm{~m}^{-2} \mathrm{~Hz}^{-1},
$$

mäßige Bursts :

$$
F=(40-200) \cdot 10^{-22} \mathrm{~W} \mathrm{~m}^{-2} \mathrm{~Hz}^{-1} \text { und }
$$

starke Bursts:

$$
F>2 \cdot 10^{-20} \mathrm{~W} \mathrm{~m}^{-2} \mathrm{~Hz}^{-1},
$$

deren Strahlungsstrom bis $5 \cdot 10^{-17} \mathrm{~W} \mathrm{~m}^{-2} \mathrm{~Hz}^{-1}$ betragen kann ${ }^{1}$.

Mit Gl. (10) folgt für die Elektronenwolken, die für schwache und mäßige Bursts verantwortlich sind, $\mu \lesssim 10^{-3}$ und für die Elektronenwolken der starken Bursts $\mu=10^{-2}-10^{-3}$. Beachtet man, daß einer Frequenz $v_{\mathrm{c}}=100 \mathrm{MHz}$, also einer Plasmafrequenz $\omega_{\mathrm{pc}}=2 \pi \cdot 10^{8} \mathrm{sec}^{-1}$, eine Elektronendichte $n_{\mathrm{c}} \approx 10^{8}$ $\mathrm{cm}^{-3}$ entspricht, so folgt für die Elektronendichte der Wolke $n_{\mathrm{w}} \approx 10^{4} \mathrm{~cm}^{-3}$ (schwache Bursts) bis $n_{\mathrm{w}} \approx 10^{6} \mathrm{~cm}^{-3}$ (sehr starke Bursts).

Mit bekanntem $\mu$ können wir unter der Voraussetzung $\vartheta \approx 1$ die spektrale Breite der in einem bestimmten Koronaniveau angeregten Plasmawellen abschätzen. Für $\mu=10^{-2}-10^{-3}$ folgt über Tab. 2 eine maximale Kreisfrequenz $\omega_{\max } \approx 1,1 \omega_{\mathrm{pc}}$. Da nach Gl. (7) die minimale Kreisfrequenz 1,0045 $\omega_{\mathrm{pc}}$ ist, ergibt sich eine natürliche Breite des Plasmawellenspektrums von etwa $10 \%$ der lokalen Plasmafrequenz.

In den obigen Betrachtungen wurde die Absorption elektromagnetischer Strahlung vernachlässigt. Das erscheint gerechtfertigt, wenn man die optischen Dicken $\tau$ für die Sonnenkorona im Bereich 20 bis $100 \mathrm{MHz}$ benutzt, die von JAEGER und WestFold ${ }^{11}$ mit dem Baumbach-Allen-Modell berechnet wurden. Danach gilt $\tau \lesssim 0,8$, sofern die Strahlung sich überhaupt in der Korona fortpflanzen kann.

\section{Gültigkeitsbereich der Intensitätenformel}

Bislang wurde (92) aus I benutzt, ohne zu berücksichtigen, daß (92) aus I nur gilt, wenn die Bedingung $E_{k} \ll 4 \pi$ e $n_{\mathrm{c}} \lambda_{\text {De }}$ [siehe I, Gl. (15); $e$ ist

10 G. Swarup, P. H. Stone u. A. Maxwell, Astrophys. J. 131, 725 [1960]. die Elementarladung] erfüllt ist. Mit (6) ergibt sich:

$$
\chi^{2}=\frac{E_{k}^{2}}{16 \pi^{2} e^{2} n_{c}^{2} \lambda_{\mathrm{Dc}}^{2}}=\frac{3,8 \cdot 10^{5}}{k \lambda_{\mathrm{Dc}}} \mu^{3} .
$$

Für einen mittleren Wert $\left(k \lambda_{\mathrm{Dc}}\right) \approx 10^{-1}$ folgt $\chi=1,9 \cdot 10^{3} \mu^{3 / 2}$ und damit $\chi=1,9$ bei $\mu=10^{-2}$ und $\chi=6 \cdot 10^{-2}$ bei $\mu=10^{-3}$. Für $\mu=10^{-2}$ ist also die Bedingung $\chi \ll 1$ nicht erfüllt, für $\mu=10^{-3} \mathrm{da}$ gegen gut. $\mathrm{Da}$ bei $\chi \gtrsim 1(92)$ aus I die Intensität unterschätzt bzw. (97) aus I mit $\sigma^{1}\left(\Omega_{1}\right) \approx 10^{-6}$ einen zu kleinen Streukoeffizienten liefert, ist zu erwarten, da dann die Glieder mit höheren Ordnungen zusätzlich beträchtliche Intensität liefern werden, deren Größe jedoch zur Zeit nicht abgeschätzt werden kann. Somit können die starken Bursts mit einem Strahlungsstrom $F>10^{-19} \mathrm{~W} \mathrm{~m}^{-2} \mathrm{~Hz}^{-1}$ bei $100 \mathrm{MHz}$ mit dem betrachteten Mechanismus nicht erklärt werden, während der Strahlungsstrom der schwachen und mäßigen Bursts eine ausreichende Erklärung findet.

Zur Prüfung der Konsistenz der obigen Betrachtungen berücksichtigen wir, daß sich nach STEwART ${ }^{7}$ die Geschwindigkeit der Elektronenwolken des Typ III über eine Strecke von 1,85 Sonnenradien (200 bis $12 \mathrm{MHz}$ ) nicht wesentlich ändert. Daher muß gelten:

$$
F 4 \pi R^{2} \Delta v D / \sigma^{1}\left(\Omega_{1}\right) \ll \frac{1}{2} m v_{0}^{2} n_{\mathrm{w}} V .
$$

Dabei ist $D$ die gesamte Burstdauer. Links steht die gesamte vom Burst abgestrahlte elektromagnetische Energie $F 4 \pi R^{2} \Delta v D$, dividiert durch den Streukoeffizienten $\sigma^{1}\left(\Omega_{1}\right)$, also die gesamte, von der Wolke infolge Anregung der Plasmawellen abgegebene Energie; rechts steht die kinetische Energie der Wolke. Für schwache und mäßige Bursts ist $F \lesssim 10^{-20} \mathrm{~W} \mathrm{~m}^{-2} \mathrm{~Hz}^{-1}$ bei $100 \mathrm{MHz}$. Daraus ergibt sich nach (10)

$$
\mu \approx 5 \cdot 10^{-4} \quad \text { bzw. } \quad n_{\mathrm{w}} \approx 5 \cdot 10^{4} \mathrm{~cm}^{-3}
$$

wegen $\mu=n_{\mathrm{w}} / n_{\mathrm{c}}$ und $n_{\mathrm{c}} \approx 10^{8} \mathrm{~cm}^{-3}$. Setzen wir wieder $\Delta v=10^{8} \mathrm{~Hz}$ und außerdem $D=10 \mathrm{sec}$ (W WLD, SMERD und $\mathrm{WEISS}^{1}$ ), so erhalten wir:

$$
F 4 \pi R^{2} \Delta v D / \sigma^{1}\left(\Omega_{1}\right)=2,8 \cdot 10^{25} \mathrm{erg}
$$

und

$$
\frac{1}{2} m v_{0}^{2} n_{\mathrm{w}} V=4,5 \cdot 10^{27} \mathrm{erg} \text {. }
$$

In diesem Falle ist also Gl. (11) erfüllt. Für starke Bursts mit $F=5 \cdot 10^{-17} \mathrm{~W} \mathrm{~m}^{-2} \mathrm{~Hz}^{-1}$ (bei $100 \mathrm{MHz}$ )

11 J. C. Jaeger u. K. C. Westrold, Australian J. Sci. Res. (A) $3,376[1950]$. 
ist nach Gl. (10) $\mu \approx 10^{-2}$, also $n_{\mathrm{w}} \approx 10^{6}$, und es folgt

und

$$
F 4 \pi R^{2} \Delta v D / \sigma^{1}\left(\Omega_{1}\right)=2 \cdot 10^{29} \mathrm{erg}
$$

$v_{0}^{2} n_{\mathrm{w}} V=9 \cdot 10^{28} \mathrm{erg}$

so daß Gl. (10) nicht mehr gilt. Auch diese Abschätzung zeigt, daß der aus Gl. (97) der Arbeit I gewonnene Streukoeffizient $\sigma^{1}\left(\Omega_{1}\right)$ mit $\approx 10^{-6}$ für die intensiven Bursts vom Typ III zu klein ist.

Außerdem können wir noch Gl. (9) an der Abhängigkeit von der Elektronendichte $n_{\mathrm{c}}$ der Korona prüfen. Wegen $v_{\mathrm{c}} \sim n_{\mathrm{c}}{ }^{1 / 2}$ und $\mu \sim n_{\mathrm{c}}{ }^{-1}$ folgt aus (10)

$$
F \sim n_{\mathrm{c}}^{-3 / 2} \quad \text { bzw. } \quad F \sim v_{\mathrm{c}}^{-3} .
$$

Den Messungen von Hughes und Harkness ${ }^{9}$ entnimmt man, daß für die Dauer $D_{v_{\mathrm{c}}}$ des Typ III bei fester Frequenz $v_{\mathrm{c}}$ grob $D_{v_{\mathrm{c}}} \sim v_{\mathrm{c}}{ }^{-1}$ gilt, so daß $F D_{v_{\mathrm{c}}} \sim v_{\mathrm{c}}{ }^{-4}$ folgt. Das Ergebnis Gl. (12) läßt sich auch mit Messungen von $\mathrm{W}_{\text {ILD }}{ }^{12}$ vergleichen, der $F D_{v_{\mathrm{c}}} \sim v_{\mathrm{c}}{ }^{-n}$ mit $2,8 \leqq n \leqq 3,6$ angibt.

Alle bisherigen Erörterungen bezogen sich nur auf die Grundfrequenz.

Aus dem Vergleich der in I hergeleiteten Formeln (63) und (92) (in I, Abschnitt IV e), ergibt sich auf Grund der zu erwartenden Plasmawellenlängen, daß die Intensität in der Oberschwingung nach (63) wesentlich geringer als in der Grundfrequenz ist. Damit kann das Auftreten eines harmonischen Bandes, dessen Intensität gleich der in der Grundfrequenz ist, mit den Formeln aus I nicht erklärt werden.

\section{Diskussion der Ergebnisse anderer Autoren}

Eine Abschätzung der spektralen Breite der in einem bestimmten Koronaniveau von der Elektronenwolke angeregten Plasmawellen wurde von MALville ${ }^{13}$ durchgeführt. Malville ging von der elektrischen Feldstärke $E_{k}$ einer infolge Zweistrominstabilität entstandenen Plasmawelle der Wellenzahl $k$ aus. Nach einer Formel von Boнm und Gross ${ }^{14}$ (im folgenden wird die Schreibweise von WiLD, Smerd und Weiss ${ }^{1}$ benutzt) gilt:

$$
E_{k}=\frac{m}{2 e k^{3}}\left\{\left.\frac{16}{3} v_{\mathrm{ph}} \omega_{\mathrm{pw}}^{2} \frac{\varrho_{\mathrm{w}}}{\varrho_{\mathrm{c}}} \frac{\partial f_{\mathrm{w}}}{\partial v}\right|_{v=v_{\mathrm{ph}}}\right\}^{2} .
$$

Dabei ist $v_{\mathrm{ph}}=\omega / k$ die Phasengeschwindigkeit der betrachteten Plasmawelle, $\varrho_{\mathrm{c}}$ die Stoßfrequenz der

12 J. P. Wild, Australian J. Sci. Res. (A) 3, 541 [1950].

13 J. M. Malville, Astrophys. J. 136, 266 [1962].

14 D. Вонм U. E. P. Gross, Phys. Rev. 75, 1864 [1949].
Elektronen der Korona, $\omega_{\mathrm{pw}}$ die Plasmafrequenz, $\varrho_{\mathrm{w}}$ die Stoßfrequenz der Elektronen, $f_{\mathrm{w}}$ die (normierte) Verteilungsfunktion der Elektronen in der Wolke. Malville ${ }^{13}$ folgerte aus (13) für die angeregten Plasmawellen eine spektrale Breite von $7 \mathrm{kHz}$ bei $25 \mathrm{MHz}$ bzw. $50 \mathrm{kHz}$ bei $200 \mathrm{MHz}$. Dabei geht der Autor offensichtlich von einer Phasengeschwindigkeit der angeregten Plasmawellen aus, die etwa gleich der Geschwindigkeit der Wolke ist. Dieser Ansatz ist jedoch nur richtig, solange die „effektive“ Geschwindigkeit $v_{0} \cos \eta \approx v_{0}$ ist, d. h. solange der Winkel $\eta$ zwischen der Fortpflanzungsrichtung der Plasmawelle und der Geschwindigkeitsrichtung der Wolke nicht zu groß wird. Letztlich setzt der Autor also voraus, daß die Plasmawellen in einem nicht zu großen Kegel um die Geschwindigkeitsrichtung der Wolke angeregt werden. Im Anschluß an Tab. 2 zeigte sich jedoch, daß diese Annahme nicht richtig ist.

Nach derselben Methode, die Gl. (12) lieferte, versuchten WiLd, Smerd und Weiss ${ }^{1}$ unter Verwendung von Gl. (13) und den Intensitätenformeln von Ginzburg und Zhelezniakov ${ }^{15}$ für die Entstehung elektromagnetischer Strahlung durch Streuung von Plasmawellen an thermischen Schwankungen die Elektronendichte der Wolke abzuschätzen. Für das elektrische Feld der beim Durchgang der Wolke durch die Korona entstehenden Plasmawellen ergibt Gl. (13) jedoch eine von Gl. (6) beträchtlich abweichende Beziehung.

Gl. (13) liefert eine zu starke Abhängigkeit des elektromagnetischen Strahlungsstromes $F$ von der Korona-Elektronendichte $n_{\mathrm{c}}$. Um das zu erkennen, setzen wir zunächst mit $\mathrm{W}_{\text {ILD }}$, SMerd und $\mathrm{W}_{\text {EIss }}{ }^{1}$

$$
f_{\mathrm{W}}(v)=\left(\pi^{1 / 2} / 2 \Delta v\right) \exp \left\{-\left[\left(v-v_{0}\right) / \Delta v\right]^{2}\right\}
$$

und

$$
\begin{gathered}
v_{\mathrm{ph}} \approx v_{0}-\Delta v \approx v_{0}, \\
\partial f_{\mathrm{w}} /\left.\partial v\right|_{v=v_{\mathrm{ph}} \approx \Delta v^{-2}} \\
\text { und } k=\omega_{\mathrm{pc}} / v_{0} .
\end{gathered}
$$

Im Gegensatz zu Gl. (3) geht $v_{0}$ an Stelle von $v_{0} \cos \eta$ in Gl. (14) ein, d. h. auf Grund des Ansatzes (14) für $f_{\mathrm{w}}$ werden auch hier wieder nur Plasmawellen in einem nicht zu großen Kegel um die Geschwindigkeitsrichtung der Wolke betrachtet. Für das elektrische Feld der Plasmawellen ergibt sich aus Gl. (13)

15 V. L. Ginzburg u. V. V. Zhelezniakov, Soviet Astron.-A.J. 2, 653 [1958]. 
mit (15), wenn $\varrho_{\mathrm{w}} / \varrho_{\mathrm{c}}=n_{\mathrm{w}} / n_{\mathrm{c}}$ gesetzt wird,

$$
\frac{E_{k}^{2}}{8 \pi} \approx 10^{2} n_{\mathrm{c}} \varkappa T_{\mathrm{c}}\left(\frac{v_{0}}{\Delta v}\right)^{8}\left(\frac{v_{0}}{v_{\mathrm{Tc}}}\right)^{2}\left(\frac{n_{\mathrm{w}}}{n_{\mathrm{c}}}\right)^{8} .
$$

Die Abhängigkeit des Strahlungsstromes $F$ (pro Hz) von $n_{\mathrm{c}}$ erhalten wir, wenn wir wieder von der Intensitätenformel (92) aus I ausgehen, (16) einsetzen und noch durch die Bandbreite $\Delta v \approx \boldsymbol{v}_{\mathrm{c}}$ der elektromagnetischen Strahlung dividieren. Wir erhalten so: $F \sim n_{\mathrm{c}}{ }^{-6,5}$, im Gegensatz zu Gl. (12). WILD, SMERD und WeIss ${ }^{1}$ erhalten dementsprechend eine Strahlungstemperatur $T_{\mathrm{b}}{ }^{\prime} \sim n_{\mathrm{c}}{ }^{-7}$. Eine Dichteänderung um den Faktor 10, also eine Frequenzänderung um den Faktor 3,16, müßte demnach eine Intensitätenänderung um $3,2 \cdot 10^{6}$ hervorrufen. In der Literatur findet sich jedoch kein Hinweis auf derart starke Intensitätenänderungen. An der Abhängigkeit $E_{k}{ }^{2}=E_{k}{ }^{2}\left(n_{\mathrm{c}}\right)$ ändert sich nichts, wenn man die Anregung von Plasmawellen in einem großen Kegel berücksichtigt. Man erhält dann

$$
\frac{E_{k}^{2}}{8 \pi} \approx 10 n_{\mathrm{c}} \varkappa T_{\mathrm{c}}\left(k \lambda_{\mathrm{Dc}}\right)^{-10}\left(\frac{\mu}{\sqrt{\vartheta}}\right)^{8} .
$$

Wie ein Vergleich mit Gl. (6) für $k \lambda_{\mathrm{Dc}}=10^{-1}$, $\vartheta=1$ zeigt, liefert Gl. (17) für $\mu>0,1$ größere, für $\mu<0,1$ kleinere Werte als Gl. (6).

Schließlich ist zu berücksichtigen, daß für die Ableitung von Gl. (13) Stöße wichtig sind, während beim Durchgang der Elektronenwolke durch die Korona Stöße unwesentlich sein werden. Denn aus Tab. 1 ergibt sich z. B. für $\mu=10^{-2}$ eine Anwachsrate $\omega_{\mathrm{i}} \approx \gamma_{\max } \omega_{\mathrm{pc}} \approx 0,5 \omega_{\mathrm{pc}}$ für die Zweistrominstabilität. Da in der Korona die Stoßzeit $\tau_{\mathrm{c}}$ für thermische Elektronen in der Größenordnung $1 \mathrm{sec}$ liegt, ist $\omega_{\mathrm{i}} \tau_{\mathrm{c}}$ sehr groß, und es ist zu erwarten, daß - bevor Stöße eine Rolle spielen - nichtlineare Effekte die Amplitude der Plasmawellen begrenzen.

\section{Zur Dispersion der Wolke}

Wir wollen noch kurz auf die Frage eingehen, inwieweit man Aussagen über $\vartheta=T_{\mathrm{w}} / T_{\mathrm{c}}$ machen kann. Diese Größe geht nicht nur in Gl. (8) und damit nach Tab. 2 in die spektrale Breite des von der Wolke angeregten Plasmawellenspektrums ein. Ein wesentlich von 1 verschiedenes $\vartheta$ würde nach Tab. 1 über

16 S. F. Smerd u. K. C. Westrold, Phil. Mag. 40, 831 [1949].

17 D. C. Montgomery u. D. A. Tidman, Plasmakinetic Theory, McGraw-Hill, New York 1964, p. 32.

18 A. Unsöld, Z. Astrophys. 50, 61 [1960]. $y=v_{0} \cos \eta\left(\sqrt{\bar{\vartheta}} v_{\mathrm{Tc}}\right)^{-1}$ das $\gamma_{\max }$ und damit die Feldstärke der angeregten Plasmawellen beeinflussen. $\vartheta$ hängt unmittelbar mit der Geschwindigkeitsdispersion der Wolke zusammen, d. h. mit der Änderung der Wolkendicke $d$ mit der Zeit. Aussagen über $d$ bekommt man aus der Bandbreite $\Delta v$ bzw. der Dauer $D_{v_{\mathrm{c}}}$ der Bursts als Funktion der Frequenz $\boldsymbol{v}_{\mathrm{c}}$. Jedoch gehen bei der Umrechnung dieser Größen in $d$ so viele Unsicherheiten ein, $\mathrm{da} ß$ eine zuverlässige Angabe der Zeitabhängigkeit von $d$ zur Zeit kaum möglich ist, und zwar aus folgenden Gründen:

1. Infolge der spektralen Breite der angeregten Plasmawellen liefert z. B. die Gl. $\left(D_{v_{\mathrm{c}}}-\tau_{0}\right) v_{0}=d$ (Hughes und Harkness ${ }^{9}$, wobei $\tau_{0}$ die Zerfallszeit der Plasmawellen in dem betreffenden Koronaniveau nach Durchgang der Wolke ist) zu große Werte von $d$. Die anzubringende Korrektur hängt von der spektralen Breite der Plasmawellen (und damit von $\mu$ und $\vartheta$ !) und dem Elektronendichteverlauf in der Korona ab.

2. Die Zeit $\tau_{0}$ wird mit der Stoßzeit $\tau_{\mathrm{c}}$ der thermischen Elektronen in der jeweiligen Koronahöhe identifiziert. Meist wird $\tau_{\mathrm{c}}$ nach einer Formel von Smerd und Westrold ${ }^{16}$ berechnet. Richtiger erscheint die aus plasmakinetischen Betrachtungen gewonnene Zeit $\tau_{\mathrm{c}}=\tau_{\mathrm{D}}$ für $90^{\circ}$-Stöße ${ }^{17}$. Danach gilt $\tau_{\mathrm{c}} \sim T_{\mathrm{c}}^{3 / 2} \nu_{\mathrm{c}}{ }^{-2} . \tau_{\mathrm{c}}$ hängt also ziemlich stark von der Koronatemperatur $T_{\mathrm{c}}$ ab, die meist $10^{6}{ }^{\circ} \mathrm{K}$ bis $2 \cdot 10^{6}$ ${ }^{\circ} \mathrm{K}$ gewählt wird. Über aktiven Gebieten kann jedoch $T_{\mathrm{c}}=(4-6) \cdot 10^{6}{ }^{\circ} \mathrm{K}\left(\mathrm{UnsöLD}^{18}\right)$ und sogar $T_{\mathrm{c}}=10^{7}{ }^{\circ} \mathrm{K}$ (ShklovskiI ${ }^{19}$ ) betragen. Diese Unsicherheit in $T_{\mathrm{c}}$ bedingt eine große Unsicherheit in $\tau_{\mathrm{c}}$.

3. Die Identifikation $\tau_{0}=\tau_{\mathrm{c}}$ ist außerdem fraglich, wenn man berücksichtigt, daß nach $\mathrm{W}_{\text {ILD }}{ }^{12}$ die Dämpfungskonstante $\tau_{\mathrm{b}}$ der Bursts - die Intensität fällt bei fester Frequenz $v_{c}$ nach Erreichen des Maximums exponentiell ab - proportional $\nu_{\mathrm{c}}{ }^{-1}$ ist, während $\tau_{\mathrm{c}} \sim \nu_{\mathrm{c}}^{-2}$ ist. Eine Bestimmung der Koronatemperatur mit Hilfe einer aus einem Zeitprofil der Bursts (Intensität gegen die Zeit bei fester Frequenz aufgetragen) gewonnenen Dämpfungszeit $\tau_{\mathrm{b}}$ über die Gleichung $\tau_{b}=\tau_{c}$ (Elgaroy und Rodberg ${ }^{20}$ ) ist aus demselben Grund ziemlich unsicher.

19 I. S. Shklovskir, Physics of the Solar Corona, Pergamon Press, New York 1965.

20 O. Elgaroy u. H. Rodberg, Astrophys. Norveg. 8, Heft 9 [1963]. 
4. Ein Vergleich der Messungen von Maxwell, Howard und Garmire ${ }^{21}$, Boischot, Lee und Warwick ${ }^{22}, W_{\text {ILD }}$ und Sheridan ${ }^{23}$, Malville $^{13}$ und Hughes und Harkness ${ }^{9}$ zeigt, daß die Werte für $D_{v_{\mathrm{c}}}$ selbst, vor allen Dingen für kleinere Frequenzen, noch ziemlich unsicher sind.

Aus diesen Gründen bleibt eine Bestimmung der Zeitabhängigkeit der Dicke $d$ der Wolke unsicher, und einer Änderung von $d$ um den Faktor 3 über

21 A. Maxwell, W. E. Howard u. G. Garmire, Sci. Rep. No. 14 [1959], AF 19 (604) - 1394, Astia.

22 A. Borschot, R. H. Lee u. J. W. W Arwick, Astrophys. J. 131, 61 [1960].
2 Sonnenradien ${ }^{9}$ kann daher keine allzu große Bedeutung zugemessen werden. Zur Zeit können also keine Aussagen über $\vartheta$ gemacht werden.

Herrn Prof. Dr. E. Richter danke ich für die Anregung zu dieser Arbeit und für viele Hinweise und Ratschläge sehr herzlich. Herrn Prof. Dr. A. UnsöLd bin ich für die Bereitstellung eines Arbeitsplatzes und für sein freundliches, förderndes Interesse zu Dank verpflichtet.

23 J. P. Wild u. K. V. Sheridan, J. Phys. Soc. Japan 17, Suppl. A-II 223 [1962].

\title{
Untersuchung über die Ausbreitung von elektromagnetischen Wellen in einem zylindrischen Plasma mit Magnetfeld
}

\author{
Karl Weinhardt \\ Institut für Plasmaphysik GmbH, Garching bei München \\ (Z. Naturforschg. 22 a, 1592-1599 [1967] ; eingegangen am 5. Juni 1967)
}

\begin{abstract}
Propagation of circularly symmetric electromagnetic modes parallel to the magnetic field lines in the positive column of an argon hollow-cathode arc discharge has been studied. The applied frequency $\left(3 \cdot 10^{9} \mathrm{cps}\right)$ was less than both the electron gyrofrequency and the electron plasma frequency. These measurements were compared with dispersion relations for circulary symmetric modes calculated by using the complete Maxwell equations, the $\varepsilon$-tensor for a cold collisionless plasma, and suitable boundary conditions. It could be shown that the mode which was excited was most likely determined by the boundary of the coupling system and not by the boundary of the whole vessel as originally expected.
\end{abstract}

Ebene elektromagnetische Wellen, die sich in einem homogenen Plasma parallel zur Richtung eines homogenen Magnetfeldes ausbreiten, mit Frequenzen unterhalb der Elektronenplasma- und Elektronengyrofrequenz, sollten sich außerordentlich gut für diagnostische Zwecke eignen. Denn die Ausbreitung dieser sog. whistler-mode ist beschränkt auf einen schmalen Kegel um die Richtung des Magnetfeldes, wobei ihr Brechungsindex immer $>1$ und in der Nähe der Gyroresonanz stark dispersiv ist ${ }^{1}$. Für die Anregung und den Nachweis dieser Wellen bieten sich, besonders in elektrodenlosen Entladungen, kurze Stiftantennen als besonders einfach an. WIE$\mathrm{DER}^{2}$ fand jedoch an ZETA, daß die Auswertung

* Auszug aus einer von der Fakultät für Allgemeine Wissenschaft der Technischen Hochschule München genehmigten Dissertation. Tag der Promotion: 27. 7. 1966.

* Die nachstehende Arbeit wurde im Rahmen des Vertrages zwischen dem Institut für Plasmaphysik $\mathrm{GmbH}$ und der Europäischen Atomgemeinschaft über die Zusammenarbeit auf dem Gebiete der Plasmaphysik durchgeführt. solcher Messungen praktisch unmöglich gemacht wurde durch Wellen, die offenbar unter Mitwirkung des Plasmarandes zustande kamen, obwohl die Plasmaausdehnungen groß gegen die Wellenlänge waren. Aber auch unter wesentlich einfacheren Versuchsbedingungen, nämlich bei Anwendung dieser Methode in der positiven Säule eines Argon-Hohlkathodenbogens, konnten wir unsere Beobachtungen nur qualitativ mit der Theorie ebener Wellen erklären. Wieder mußte angenommen werden, daß die Stiftantennen in unkontrollierbarer Weise mehrere Wellentypen anregten, die sich unter Mitwirkung des Plasmarandes ausbreiteten und interferierten ${ }^{3}$. Es lag deshalb nahe, unser Experiment so abzu-

1 D. W. Mahaffey, Phys. Rev. 129, 1481 [1963]. - A. N. Dellis u. J. M. Weaver, Proc. Phys. Soc. London 83, 473 [1964].

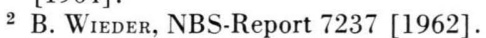

${ }^{3} \mathrm{~K}$. Weinhardt, Bericht IPP 2/59. 